ETH-TH/92-14

March 1992

hepth@xxx/9203074

\title{
A cubic action for self-dual Yang-Mills
}

\author{
ANDREW PARKES \\ Theoretische Physik, \\ ETH-Hönggerberg, \\ 8093 Zürich, \\ Switzerland.
}

\begin{abstract}
We make a change of field variables in the $\mathrm{J}$ formulation of self-dual Yang-Mills theory. The field equations for the resulting algebra valued field are derivable from a simple cubic action. The cubic interaction vertex is different from that considered previously from the $\mathrm{N}=2$ string, however, perturbation theory with this action shows that the only non-vanishing connected scattering amplitude is for three external particles just as for the string.
\end{abstract}




\section{INTRODUCTION}

Over the years self-dual Yang-Mills (SDYM) theory has received a great deal of attention. The majority of this attention has focused on finding and classifying exact solutions of the classical equations of motion, or using the self-dual solutions to find quantum properties of the Yang-Mills action. For this kind of work there is no need to derive the self-duality equations themselves from an action. However, as realised by Ooguri and Vafa [1], SDYM and self-dual gravity (SDG) in $2+2$ dimensions are the effective field theory of the $\mathrm{N}=2$ string. Consistency of the string theory implies that these self-dual systems have the remarkable property that (at least at tree level [1] and one-loop [2]) on-shell connected scattering amplitudes vanish for four or more legs. If we wish to do perturbation theory of SDYM then this gives a big incentive to derive the self-duality equations themselves from an action.

For SDG the equation of motion is the Plebanski equation which follows from a simple cubic action. However, for SDYM the situation is not so straightforward. Using the $\mathrm{J}$ formulation combined with an particular parametrisation of the gauge group it is possible to write an action for SDYM [3-5], and indeed this action has a correctly vanishing four point amplitude [6]. Alternatively it is possible to [8] avoid the group parametrisation and obtained an action for the $\mathrm{J}$ formulation by adding an extra dimension and using a generalisation of the Wess-Zumino-Novikov-Witten (WZNW) theory [7,8]. The resulting action is non-polynomial and whilst not an intrinsic problem this does rather contrast with with the polynomial SDG, and is a strange reversal from the usual properties of gravity and Yang-Mills actions.

Actions based on a very different approach were given in refs. [9,10]; harmonic space techniques were used in order to retain the Lorentz covariance that is lost in the $\mathrm{J}$ formulation. However, in [10] they find that the theory exhibits no scattering whatsoever, and so does not correspond to the $\mathrm{N}=2$ string or the $\mathrm{J}$ formulation which do at least have non-trivial three particle scattering.

In this paper we will show that there is a "Bäcklund" transformation from 
the J-field of SDYM to a an algebra valued field, $\Phi$. The main advantage is that the $\Phi$ equation of motion follows from a simple cubic action. We explore tree level perturbation theory based on this action and show that $\geq 4$ leg amplitudes vanish exactly as for the $\mathrm{N}=2$ string. However the cubic vertex is not the same as that from the open $\mathrm{N}=2$ string. We then consider Lorentz transformations and so illuminate the origin of our action; it can be considered as arising from the J-formulation in an infinite momentum frame. Similar methods allow us to find generalisations of the Plebanski action. We have explicitly checked the vanishing of the 4,5 and 6 leg amplitudes for all the cubic actions and so can be confident they have the properties of the $\mathrm{N}=2$ string. Mostly we work in flat space and only briefly consider the generalisation to non-trivial spaces.

\section{The CUBIC ACtion}

Initially we work with analytic fields over complexified $2+2$ space-time with a complex gauge group $G_{C}$ and Lie algebra $g_{C}$. Coordinates are denoted by $x^{\alpha}=\left(x^{\mu}, x^{\bar{\mu}}\right)$ where $x^{\mu}=(y, z)$ and $x^{\bar{\mu}}=(\bar{y}, \bar{z})$ are independent (not complex conjugates). We take $\epsilon^{y z}=\epsilon_{z y}=\epsilon^{\bar{z} \bar{y}}=\epsilon_{\bar{y} \bar{z}}=1$ and $\epsilon_{y z \bar{y} \bar{z}}=\epsilon_{y z} \epsilon_{\bar{y} \bar{z}}$ (note a change of sign with respect to ref. [6]). We also take the metric to be given by $d s^{2}=g_{\alpha \beta} d x^{\alpha} d x^{\beta}=2 g_{\mu \bar{\mu}} d x^{\mu} d x^{\bar{\mu}}=$ and define $g=\operatorname{det}\left(g_{\mu \bar{\mu}}\right)$. Taking a torsion free connection, and coupling constant $e$, the Yang-Mills field strength is $F_{\alpha \beta}=$ $\left[\partial_{\alpha}+e A_{\alpha}, \partial_{\beta}+e A_{\beta}\right]$ and satisfies the self-duality condition

$$
F_{\alpha \beta}=+\frac{1}{2} g \epsilon_{\alpha \beta \gamma \delta} F^{\gamma \delta}
$$

if and only if

$$
\epsilon^{\bar{\mu} \bar{\nu}} F_{\bar{\mu} \bar{\nu}}=0 \quad g^{\mu \bar{\mu}} F_{\mu \bar{\mu}}=0 \quad \epsilon^{\mu \nu} F_{\mu \nu}=0
$$

We now restrict to trivial topology and a flat metric given by $\eta_{\mu \bar{\mu}} d x^{\mu} d x^{\bar{\mu}}=d y d \bar{y}-$ $d z d \bar{z}$. Then, (2) implies that there exist $D \in G_{C}$ and $\bar{D} \in G_{C}$ such that $e A_{\mu}=$ 
$D^{-1} \partial_{\mu} D$ and $e A_{\bar{\mu}}=\bar{D}^{-1} \partial_{\bar{\mu}} \bar{D}$. Defining $J=D \bar{D}^{-1}$ reduces the duality conditions to the following well known field equation for $\mathrm{J}$

$$
\eta^{\mu \bar{\mu}} \partial_{\bar{\mu}}\left(J^{-1} \partial_{\mu} J\right)=0
$$

At this point we are motivated by the similarity to the chiral model in $1+1$ spacetime and its reformulation as discussed for example in ref. [11]. Defining $B_{\mu}$ by

$$
e B_{\mu}=J^{-1} \partial_{\mu} J
$$

we see that given a solution of (3) then, generically, there exists some $\Phi \in g_{C}$ such that

$$
B_{y}=\partial_{\bar{z}} \Phi \quad B_{z}=\partial_{\bar{y}} \Phi
$$

This automatically solves (3). Finally (4) implies $\left[\partial_{y}+e B_{y}, \partial_{z}+e B_{z}\right]=0$ which yields the following field equation for $\Phi$

$$
\eta^{\mu \bar{\mu}} \partial_{\mu} \partial_{\bar{\mu}} \Phi+e \epsilon^{\bar{\mu} \bar{\nu}} \partial_{\bar{\mu}} \Phi \partial_{\bar{\nu}} \Phi=0
$$

This equation appeared in ref. [12] as part of more general study of Bäcklund transformations in SDYM. For us the point of this construction is that (6) is the Euler-Lagrange equation of the following action

$$
S=\frac{1}{C_{R}} \operatorname{Tr} \int d^{4} x\left[-\frac{1}{2} \eta^{\mu \bar{\mu}} \partial_{\mu} \Phi \partial_{\bar{\mu}} \Phi+\frac{e}{3} \epsilon^{\bar{\mu} \bar{\nu}} \partial_{\bar{\mu}} \Phi \partial_{\bar{\nu}} \Phi \Phi\right]
$$

where as usual $\Phi=\Phi^{a} T_{a},\left[T_{a}, T_{b}\right]=f_{a b c} g^{c d} T_{d}$ and $\operatorname{Tr}\left(T_{a} T_{b}\right)=-C_{R} g_{a b}$.

In the above we have used the $\mathrm{J}$ formulation only in order to exhibit the gauge independence of the construction. A more direct route is to make the Yang-Mills gauge choice $A_{\bar{\mu}}=0$ in which case $B_{\mu}=A_{\mu}$ and then (5) follows as the general solution of $\eta^{\mu \bar{\mu}} F_{\mu \bar{\mu}}=0$. Finally $F_{y z}=0$ gives (6) as the field equation for $\Phi$. 
Conversely, given a field $\Phi$ then we use (5) to define fields $B_{\mu}$ which automatically satisfy the "conservation law"

$$
\eta^{\mu \bar{\mu}} \partial_{\bar{\mu}} B_{\mu}=0
$$

The field equation for $\Phi$ then implies we can find a $J \in G_{C}$ such that (4) holds true, and so we recover (3). In particular, $A_{\mu}=B_{\mu}$ with $A_{\bar{\mu}}=0$ gives a self-dual Yang-Mills field strength.

We have seen that (5) maps solutions of (3) to solutions of (6) and vice versa. It is also a Bäcklund transformation between two fields in the sense that consistency of the transformation implies that both fields must satisfy their equations of motion. Thus, we only have shown equivalence of the on-shell theories; however the viewpoint in this paper shall be to investigate (7) for both on and off-shell fields.

In order to have four real dimensions and a real gauge group we must impose some reality conditions. The standard choice is to take $\left(x^{\mu}\right)^{*}=x^{\bar{\mu}},\left(A_{\mu}\right)^{\dagger}=$ $-A_{\bar{\mu}}$ and $J^{\dagger}=J$, but in this case the Bäcklund transformation is generally not compatible with a simple reality condition on $\Phi$. It is much simpler to implement the reality condition by $\left(x^{\alpha}\right)^{*}=x^{\alpha}$ and $A_{\alpha}^{\dagger}=-A_{\alpha}$. In this case $J \in G_{R}$ and $\Phi^{\dagger}=-\Phi \in g_{R}$. Notice that if we want a real gauge group then we are forced to take a $2+2$ space-time; this method cannot treat $\mathrm{SU}(2)$ instantons in Euclidean space.

\section{Perturbation theory}

A striking property of the $\mathrm{N}=2$ string and so of these self-dual systems is that in tree level perturbation theory the on shell connected Feynman diagrams sum to zero for four or more external legs. For consistency it should be that the perturbation theory based on (7) should have the same property. 
It is very convenient to define

$$
\begin{gathered}
a_{i j}=\epsilon^{\mu \nu} k_{\mu i} k_{\nu j} \quad \bar{a}_{i j}=\epsilon^{\bar{\mu} \bar{\nu}} k_{\bar{\mu} i} k_{\bar{\nu} j} \\
k_{i j}=\eta^{\mu \bar{\nu}} k_{\mu i} k_{\bar{\nu} j} \quad s_{i j}=k_{i j}+k_{j i} \quad c_{i j}=k_{i j}-k_{j i}
\end{gathered}
$$

From the action (7) we have the usual propagator $\left\langle\Phi^{a}(k) \Phi^{b}(-k)\right\rangle \propto g^{a b} / k^{2}$ whilst our off-shell three leg vertex is simply $<\Phi^{a}\left(k_{1}\right) \Phi^{b}\left(k_{2}\right) \Phi^{c}\left(-k_{1}-k_{2}\right)>\propto$ $e \bar{a}_{12} f_{a b c}$. We emphasise that the three leg on-shell ampliude is not identically zero and so we do not have the same theory as in ref. [10]. or comparison the $\mathrm{N}=2$ string gives a vertex proportional to $c_{12}$ instead of $\bar{a}_{12}$. We can generate many identities by taking the trivial identity

$$
k_{\mu i} k_{\nu j} k_{\rho m}+\operatorname{antisym}(\mathrm{ijm}) \equiv 0
$$

and contracting the free space-time indices with various tensors. For example, we find

$$
\bar{a}_{i j} k_{m n}+\operatorname{cycl}(\mathrm{ijn}) \equiv 0
$$

which leads to

$$
k_{i i}=\Sigma_{1}^{4} k_{i}=0 \quad \Longrightarrow \quad \frac{\bar{a}_{12} \bar{a}_{34}}{s_{12}}+\frac{\bar{a}_{13} \bar{a}_{24}}{s_{13}} \equiv 0
$$

This can easily be used to show the vanishing of the on-shell tree-level four leg amplitude without the need for a four leg vertex. The corresponding "cc/s" identities have extra terms which signify the need for a four leg vertex, as in perturbation theory based directly on the $\mathrm{J}$ formulation.

Above 4 legs I found it much better not to try to derive more identities but simply to apply the much more inelegant brute-force (and ideal for computer algebra) technique of solving for the components of momenta so as to put all external 
legs on shell. In this way I have been able to verify that the action (7) leads to vanishing of the on-shell tree-level 5 and 6 leg diagrams. It is important to go to at least 6 legs because only then do we fully probe the off-shell structure of the vertex because with 4 and 5 external legs all diagrams are such that at least one leg of every vertex is on shell. For the same reason I expect that if the 4,5, and 6 leg amplitudes vanish then we can be confident that all tree-level amplitudes with more legs will also vanish.

At one loop loop it is easy to see that the tadpole and propagator corrections vanish both on- and off-shell. The three point amplitude is propartio9nal to $\bar{a}_{12}{ }^{3}$ and is both UV and IR divergent. Thus, as expected the theory is not renormalisable. In the string version the UV divergence is removed by the modular invariance leaving just IR divergences (as pointed out in [1,2]) and it is interesting to speculate that some kind of "soft-photon" sum could remove all these as well.

\section{Reduction to two dimensions}

Having seen that the action (7) has the expected properties in tree level perturbation theory it is natural to further explore its properties by looking at two dimensional version.

Suppose we trivially reduce by forcing fields to depend only on $\eta_{i}=k_{\mu i} x^{\mu}+$ $k_{\bar{\mu} i} x^{\bar{\mu}}$ with $i=1,2$ and $k_{11}=k_{22}=0$. Then the the J equation (3) becomes

$$
k_{12} \partial_{1}\left(J^{-1} \partial_{2} J\right)+k_{21} \partial_{2}\left(J^{-1} \partial_{1} J\right)=0
$$

which is simply the equation of motion of the principal chiral model with a WessZumino term proportional to $c_{12}$. It becomes a WZNW theory at $k_{12}=0$ or $k_{21}=0$.

The Bäcklund transformation (4),(5) reduces to

$$
\left(\begin{array}{ll}
k_{y 1} & k_{y 2} \\
k_{z 1} & k_{z 2}
\end{array}\right)\left(\begin{array}{c}
J^{-1} \partial_{1} J \\
J^{-1} \partial_{2} J
\end{array}\right)=\left(\begin{array}{ll}
k_{\bar{z} 1} & k_{\bar{z} 2} \\
k_{\bar{y} 1} & k_{\bar{y} 2}
\end{array}\right)\left(\begin{array}{c}
\partial_{1} \Phi \\
\partial_{2} \Phi
\end{array}\right)
$$

The two matrices involved are both non-singular only if $a_{12} \bar{a}_{12} \neq 0$ and from 
the identity $a_{12} \bar{a}_{12}=k_{11} k_{22}-k_{12} k_{21}$ we see this needs $k_{12} k_{21} \neq 0$. Thus, the transformation fails precisely in the case in which the model is a WZNW model. However, away from this singular point we can proceed to do the transformation. In this case (6) reduces to

$$
k_{12} \partial_{1} \partial_{2} \Phi+e \bar{a}_{12}\left[\partial_{1} \Phi, \partial_{2} \Phi\right]=0
$$

coming from the reduced action

$$
S=\frac{1}{C_{R}} \operatorname{Tr} \int d^{2} \eta\left[-\frac{s_{12}}{2} \partial_{1} \Phi \partial_{2} \Phi-\frac{e \bar{a}_{12}}{3}\left[\partial_{1} \Phi, \partial_{2} \Phi\right] \Phi\right]
$$

This provides an action for the the chiral model with Wess-Zumino term in $1+1$ dimensions that does not require any explicit parametrisation of the group nor an extension into 3 dimensions. The action differs from the one without Wess-Zumino term only in a rescaling of the coupling constant. It may also be a disadvantage that the transition to algebra rather than group valued fields loses a lot of the group structure. The transition itself is only a local construction. Despite this it would be interesting to look at the $1+1$ dimensional quantum theory based on $(17)$.

Alternatively we can solve (14) by writing

$$
J^{-1} \partial_{1} J=k_{12} \partial_{1} P \quad J^{-1} \partial_{2} J=-k_{21} \partial_{2} P
$$

and the identical construction leads to the action

$$
S=\frac{1}{C_{R}} \operatorname{Tr} \int d^{2} \eta\left[-\frac{s_{12}}{2} a \partial_{1} P \partial_{2} P-\frac{k_{12} k_{21}}{3}\left[\partial_{1} P, \partial_{2} P\right] P\right]
$$

Ignoring the fact that (18) is singular than the theory is clearly classically trival at the WZNW case. 


\section{LORENTZ SYMMETRY}

Our starting point was the self-duality equation, (1), which posseses a manifest $\mathrm{SO}(2,2)$ Lorentz symmetry. However, both the $\mathrm{J}$ formulation and the formulation given above obscure this symmetry by "solving" (1) in a non-covariant fashion. In

particular, we chose a symplectic structure when we made a split into $x^{\mu}$ and $x^{\bar{\mu}}$ and did not treat them equally. The remaining manifest space-time symmetry of (19) with real coordinates is a meagre $\mathrm{SL}(2, \mathrm{R})$ (in comparison, the J formulation in a $\mathrm{C}^{1,1}$ space has the more inviting $\mathrm{U}(1,1)$ symmetry). Despite this noncovariance it is still instructive to consider the effects of an $\mathrm{SO}(2,2)$ Lorentz transformation on (19) and so vary the symplectic structure within $\mathrm{SO}(2,2) / \mathrm{SL}(2, \mathrm{R})$. We consider

$$
\begin{gathered}
\partial_{\mu} \rightarrow f_{1} \partial_{\mu}-f_{2} \epsilon_{\mu \nu} \eta^{\nu \bar{\nu}} \partial_{\bar{\nu}} \\
\partial_{\bar{\mu}} \rightarrow \bar{f}_{1} \partial_{\bar{\mu}}-\bar{f}_{2} \epsilon_{\bar{\mu} \bar{\nu}} \eta^{\nu \bar{\nu}} \partial_{\nu}
\end{gathered}
$$

where $f_{i}$ and $\bar{f}_{i}$ are constant parameters subject to the restriction $\left(f_{1} \bar{f}_{1}+f_{2} \bar{f}_{2}\right)=1$ in order that $\eta^{\mu \bar{\mu}} \partial_{\mu} \partial_{\bar{\mu}}$ is invariant.

Under such a transformation the action (19) is not invariant but becomes

$$
\begin{aligned}
S= & \frac{1}{C_{R}} \operatorname{Tr} \int d^{4} x\left[-\frac{1}{2} \eta^{\mu \bar{\mu}} \partial_{\mu} \Phi \partial_{\bar{\mu}} \Phi\right. \\
& \left.+\frac{e}{3}\left(\bar{f}_{1}^{2} \epsilon^{\bar{\mu} \bar{\nu}} \partial_{\bar{\mu}} \Phi \partial_{\bar{\nu}} \Phi \Phi+\bar{f}_{1} \bar{f}_{2} \eta^{\mu \bar{\mu}}\left[\partial_{\mu} \Phi, \partial_{\bar{\mu}} \Phi\right] \Phi+\bar{f}_{2}^{2} \epsilon^{\mu \nu} \partial_{\mu} \Phi \partial_{\nu} \Phi \Phi\right)\right]
\end{aligned}
$$

and the momentum part of the corresponding three point vertex is $\bar{f}_{1}^{2} \bar{a}_{12}+\bar{f}_{1} \bar{f}_{2} c_{12}+$ $\bar{f}_{2}^{2} a_{12}$ Again, explicit calculations show that the tree-level 4,5 and 6 point scattering amplitudes vanish. This is not surprising since we have only simply done a global space-time boost.

It is also possible to use this procedure to generate "new" actions for self-dual gravity which have the same properties for the scattering amplitudes. Consider 
the generalised Plebanski action $S=\int d^{4} x L$ with

$$
\begin{aligned}
L= & \frac{1}{2} \eta^{\mu \bar{\mu}} \partial_{\mu} \varphi \partial_{\bar{\mu}} \varphi \\
& +e_{a a} \epsilon^{\mu \nu} \epsilon^{\rho \sigma} \partial_{\mu} \partial_{\rho} \varphi \partial_{\nu} \partial_{\sigma} \varphi \varphi+2 e_{c a} \epsilon^{\mu \nu} \eta^{\rho \bar{\sigma}} \partial_{\mu} \partial_{\rho} \varphi \partial_{\nu} \partial_{\bar{\sigma}} \varphi \varphi \\
& +2 e_{a \bar{a}} \epsilon^{\mu \nu} \epsilon^{\bar{\mu} \bar{\nu}} \partial_{\mu} \partial_{\bar{\mu}} \varphi \partial_{\nu} \partial_{\bar{\nu}} \varphi \varphi \\
& +2 e_{c c} \eta^{\mu \bar{\nu}} \eta^{\rho \bar{\sigma}}\left(\partial_{\mu} \partial_{\rho} \varphi \partial_{\bar{\nu}} \partial_{\bar{\sigma}} \varphi \varphi-\partial_{\mu} \partial_{\bar{\sigma}} \varphi \partial_{\bar{\nu}} \partial_{\rho} \varphi \varphi\right) \\
& +2 e_{c \bar{a}} \epsilon^{\bar{\mu} \bar{\nu}} \eta^{\rho \bar{\sigma}} \partial_{\bar{\mu}} \partial_{\rho} \varphi \partial_{\bar{\nu}} \partial_{\bar{\sigma}} \varphi \varphi \\
& +e_{\bar{a} \bar{a}} \epsilon^{\bar{\mu} \bar{\nu}} \epsilon^{\bar{\rho} \bar{\sigma}} \partial_{\bar{\mu}} \partial_{\bar{\rho}} \varphi \partial_{\bar{\nu}} \partial_{\bar{\sigma}} \varphi \varphi
\end{aligned}
$$

The usual Plebanski equation corresponds to the case where only $e_{a \bar{a}}$ is non-zero. This gives rise to a three point vertex proportional to

$$
e_{a a} a_{12}^{2}+e_{c a} c_{12} a_{12}+e_{a \bar{a}} a_{12} \bar{a}_{12}+e_{c c} c_{12}^{2}+e_{c \bar{a}} c_{12} \bar{a}_{12}+e_{\bar{a} \bar{a}} \bar{a}_{12}^{2}
$$

and by explicit calculation I find that the on-shell 4 and 5 point scattering amplitudes vanish if

$$
\begin{array}{r}
e_{a a} e_{\bar{a} \bar{a}}-e_{c a} e_{c \bar{a}}+e_{a \bar{a}} e_{c c}+e_{c c}^{2} \equiv 0 \\
\left(e_{c \bar{a}}^{2}-4 e_{c c} e_{\bar{a} \bar{a}}\right)\left(2 e_{a \bar{a}} e_{\bar{a} \bar{a}}-e_{c \bar{a}}{ }^{2}-\left(e_{c \bar{a}}^{2}-4 e_{c c} e_{\bar{a} \bar{a}}\right)\right)^{2}- \\
\left(4 e_{\bar{a} \bar{a}}^{2} e_{c a}-2 e_{a \bar{a}} e_{\bar{a} \bar{a}} e_{c \bar{a}}+2 e_{c \bar{a}}\left(e_{c \bar{a}}{ }^{2}-4 e_{c c} e_{\bar{a} \bar{a})}\right)^{2} \equiv 0\right.
\end{array}
$$

I write the conditions in this form because in this way they are most convenient for the computer calgebra calculations. I found it best to solve the first condition for $e_{a a}$ and then to introduce $\tilde{e}_{c \bar{a}}$ by setting $e_{c c}=\left(e_{c \bar{a}}^{2}-\tilde{e}_{c \bar{a}}^{2}\right) /\left(4 e_{\bar{a} \bar{a}}\right)$ and solving the second condition for $e_{c a}$. In this fashion I have managed to do the 6 point function with random numeric values for the on-shell incoming momenta and find the graphs always sum to zero. So with the conditions (24) the tree-level 6 particle amplitude also vanishes on-shell.

In fact this solution arises simply from applying the transformation (20) to the 
original Plebanski action; we get

$$
\begin{array}{lrl}
e_{a a} & =f_{1}^{2} \bar{f}_{2}^{2} & e_{c a}=\bar{f}_{1} \bar{f}_{2} f_{1}^{2}-\bar{f}_{2}^{2} f_{1} f_{2} \quad e_{c c}=-\bar{f}_{1} \bar{f}_{2} f_{1} f_{2} \\
e_{a \bar{a}}=\bar{f}_{1}^{2} f_{1}^{2}+\bar{f}_{2}^{2} f_{2}^{2} & e_{c \bar{a}}=-\bar{f}_{1}^{2} f_{1} f_{2}+\bar{f}_{1} \bar{f}_{2} f_{2}^{2} \quad e_{\bar{a} \bar{a}}=\bar{f}_{1}^{2} f_{2}^{2}
\end{array}
$$

Lorentz boosts change the form of the action but not the property that scattering amplitudes vanish for more than three legs. Thus, it is natural to ask what would happen if we did such boosts starting from the $\mathrm{J}$ formulation rather that (19). For simplicity, consider the special case that $f_{1}=\bar{f}_{1}=1, \bar{f}_{2}=0$ and $f_{2}$ is arbitrary. Then in the $\mathrm{J}$ formulation perturbation theory the cubic vertex is $e_{\mathrm{J}} c_{12} f_{a b c}$ which gets boosted to

$$
e_{\mathrm{J}}\left(f_{2}^{2} \bar{a}_{12}+c_{12}\right) f_{a b c}
$$

Hence the interaction term of (19) is naturally generated from the J formulation. Furthermore, suppose we go to an "infinite momentum frame" by taking $f_{2} \rightarrow \infty$, but simultaneously taking $e_{\mathrm{J}} \rightarrow 0$ in such a way that $e_{\mathrm{J}} f_{2}^{2}=e$ remains finite (and small). Then in this limit the $e_{\mathrm{J}} c_{12}$ cubic vertex vertex disappears. The $\mathrm{J}$ formulation also has vertices with more legs but these necessarily come with higher powers of $e_{\mathrm{J}}$ and so also vanish in the above limit. The only remaining vertex is the $e \bar{a}_{12}$ vertex that one would get from (19). Thus we can interpret (19) as arising from the $\mathrm{J}$ formulation in an "infinite momentum frame", and in the process we have reduced a non-polynomial action to a cubic action.

Bye the same process we can convert the usual action for the Plebanski equation (which is (22) with only $e_{a \bar{a}}$ non-zero) to an action in which only $e_{\bar{a} \bar{a}}$ is nonzero. Such an action solves (24) and so has vanishing scattering amplitudes for 4 or more legs.

Finally, consider a boost of the cubic action (19) with the parameters $f_{1} \bar{f}_{1}=1$, $f_{2}=\bar{f}_{2}=0$ and $\bar{f}_{1}$ arbitrary. It is easy to see that the only effect that this has is 
the rescaling of the coupling constant

$$
e \rightarrow e \bar{f}_{1}^{2}
$$

This means that if we have a solution to (6) at coupling $e$ then we can generate another solution but at coupling $e \bar{f}_{1}^{2}$ by means of such a Lorentz boost. However, if we now restrict our attention only to Lorentz and gauge invariant features of the theory, one might expect that we can than undo the Lorentz boost without changing the coupling. Thus Lorentz invariant sacattering features seem to be the same for all couplings, and so the same as in the linearised theory. Matching the often repeated statement that there is no scattering on SDYM [13]. Clearly there is scattering, but possibly not of any Lorentz invariant quantity. Indeed "correcting" the loss of manifest Lorentz invariance by use of harmonic space techniques also ended up with vanishing scattering amplitudes [10].

The $\mathrm{N}=2$ string vertex is definitely not Lorentz invariant; it could well be that any attempt to enforce such Lorentz symmetry is doomed to bring along with it triviality. We also note that SDYM is an integrable theory without a trivial Smatrix and so has somehow avoided the Coleman-Mandula theorem [14]. There are two obvious escape routes; the theorem assumes Lorentz invariance and that there is non-trivial scattering for most incoming momenta. The $\mathrm{N}=2$ string evades on both these counts. Attempting to re-instate Lorentz invariance is difficult because of the scarcity of candidate Lorentz invariant momentum dependent on-shell three point functions.

\section{Generalisation to Curved space}

The previous considerations for SDYM were for a flat background space-time. In this section we do the same things but starting with an background metric $g_{\mu \bar{\mu}}$ which satisfies the "Kähler" conditions

$$
\epsilon^{\mu \nu} \partial_{\mu} g_{\nu \bar{\nu}}=\epsilon^{\bar{\mu} \bar{\nu}} \partial_{\bar{\mu}} g_{\nu \bar{\nu}}=0
$$


We assume that it is possible to go to the gauge $A_{\bar{\mu}}=0$ then $g^{\mu \bar{\mu}} F_{\mu \bar{\mu}}=0$ becomes

$$
g^{\mu \bar{\mu}} \partial_{\bar{\mu}} A_{\mu}=0
$$

which is solved by

$$
A_{\mu}=\epsilon_{\mu \nu} g^{\nu \bar{\nu}} \partial_{\bar{\nu}}(\sqrt{-g} \Phi)
$$

where we have used $\partial_{\bar{\mu}}\left(g g^{\mu \bar{\mu}}\right)=0$ and the $1 / \sqrt{-g}$ is inserted so that $\Phi$ transforms as a scalar (rather than scalar density).

The remaining condition $F_{y z}=0$ then gives

$$
\partial_{\mu}\left[g^{\mu \bar{\mu}} \partial_{\bar{\mu}}(\sqrt{-g} \Phi)\right]-\frac{e}{g} \epsilon^{\bar{\mu} \bar{\nu}} \partial_{\bar{\mu}}(\sqrt{-g} \Phi) \partial_{\bar{\nu}}(\sqrt{-g} \Phi)=0
$$

In order to be able to derive this from an action we impose conditions on the background metric. In particular we take $\partial_{\alpha} g=0$. This is a reasonable condition to take because it makes the Riemann curvature tensor of the background self-dual and explained in ref. [1] it gives rise to the Plebanski equation and cubic action discussed earlier. When $g=\operatorname{det}\left(g_{\mu \bar{\mu}}\right)$ is constant then (30) follows from the action

$$
S=\frac{1}{C_{R}} \operatorname{Tr} \int d^{4} x(-g)\left[+\frac{1}{2} g^{\mu \bar{\mu}} \partial_{\mu} \Phi \partial_{\bar{\mu}} \Phi+\frac{e}{3 \sqrt{-g}} \epsilon^{\bar{\mu} \bar{\nu}} \partial_{\bar{\mu}} \Phi \partial_{\bar{\nu}} \Phi \Phi\right]
$$

which reduces to (19) in flat space.

Of course the above construction heavily uses the fact that our space-time is topologically trivial and requires more care in non-trivial topologies. 


\section{Conclusions}

We have shown how to describe SDYM by means of a cubic action, and that the resulting action gives scattering amplitudes with similar properties to the $\mathrm{N}=2$ string. It would certainly be interesting to explore the quantum theory of this action further, despite the fact that naive power counting suggests it is nonrenormalizable.

Finally we remark that the action for SDG of Giveon and Shapere [15] has similarities to (31) and it would certainly be interesting to couple them together. Presumably this would correspond to unravelling the geometry of the heterotic $N=2$ string discussed in ref. [1].

Acknowledgements: I am grateful to J. Schiff for drawing my attention to ref. [12]. I thank the ETH for hospitality during my fellowship. I am also grateful to

the people of Great Britain and Switzerland for their financial support through the Royal Society of Great Britain and the Swiss National Science Foundation, respectively.

\section{References}

[1] H. Ooguri and C. Vafa, Mod. Phys. Lett. A5 (1990) 1389; Nucl. Phys. B 369 (1991) 469; Nucl. Phys. B 367 (1991) 83

[2] M. Bonini, E. Gava and R. Iengo, Mod. Phys. Lett. A6 (1991) 795

[3] C.N. Yang, Phys. Rev. Lett. 38 (1977) 1377

[4] Y. Brihaye, D.B. Fairlie, J. Nuyts and R.G. Yates, J. Math. Phys. 19 (1978) 2528

[5] K. Pohlmeyer, Commun. Math. Phys. 72 (1980) 37

[6] Andrew Parkes, hepth@xxx/9110075, ETH-TH/91-35, Nucl. Phys. B, to be published 
[7] S.K. Donaldson, Proc. London. Math. Soc. 50 (1985) 1

[8] V.P. Nair and J. Schiff, Phys. Lett. B 246 (1990) 423;

Kähler-Chern-Simons theory and symmetries of anti-self-dual gauge fields, CU-TP-521 (May 1991);

V.P.Nair, Kähler-Chern-Simons Theory, hepth@xxx/9110042

[9] S. Kalitzin and E. Sokatchev, Phys. Lett. B 262 (1991) 444

[10] Neil Marcus, Yaron Oz and Shimon Yankielowicz, Harmonic space, self-dual Yang-Mills and the $N=2$ string, hepth@xxx/9112010, TAUP-1928-91 (Dec 1991)

[11] V.E. Zakharov and A.V. Mikhailov, Sov. Phys. JETP 47 (1978) 1017

[12] M. Bruschi, D. Levi and O. Ragnisco, Lett. Nuov. Cim. 33 (1982) 263

[13] R.S. Ward, The twistor approach to differential equations, in Quantum Gravity 2, ed. C.J. Isham, R. Penrose and D.W. Sciama (Oxford Univ. Press 1981)

[14] S. Coleman and J. Mandula, Phys. Rev. D 159 (1967) 1251

[15] Amit Giveon and Alfred Shapere, Gauge symmetries of the $N=2$ string, hepth@xxx/9203008, IASSNS-HEP-92-14 